\title{
A Comprehensive Analysis of MEMS Electrothermal Displacement Sensors
}

\author{
Ali Bazaei, Member, IEEE, and S. O. Reza Moheimani, Fellow, IEEE
}

\begin{abstract}
For electrothermal microelectromechanical system position sensors, we introduce a novel analytical model that captures the nonuniform distribution of temperature as well as the nonlinear dependence of resistivity on temperature. The proposed model also captures the effects of contoured beam heaters and the nonuniformity of the air gap between the heatsink and the heaters, which varies with heat-sink position and is differentially transduced into the output voltage. The model accurately predicts the experimentally obtained $I-V$ data and the corresponding sensor output. It also explains the considerable improvement achieved in the linearity of the sensor response when the beam profiles are appropriately shaped to yield a more uniform temperature distribution. The shaped sensor is compared with conventional uniform electrothermal sensors under two different operating conditions, voltage, and current bias modes. Improved linearity is observed in both cases. The model is also applicable to predict the dynamic response of the sensor. An iterative procedure is developed to solve the additional complexity in voltage mode, which is a nonlinear partial integro-differential equation. Considering different bias modes and heater profiles, we evaluate the sensor bandwidth and linearity using the model and conduct experiments to validate the results. Based on first principles, the proposed model is more transparent than sophisticated software-based approaches and compatible with traditional solvers in MATLAB.
\end{abstract}

Index Terms-Electrothermal, position sensor, MEMS, nonlinear analysis.

\section{Motivation}

$\mathbf{I}$ N MANY micro-electro-mechanical systems (MEMS), it is highly desirable to measure the displacement of moving components accurately [1]-[8]. This is particularly important in applications such as probe-based data storage and scanningprobe microscopy, where the required high positioning accuracies are achieved by feedback control and displacement sensing [9]-[13]. Among the position sensors that can be easily fabricated in MEMS, the electrothermal sensors have the smallest footprint and a large dynamic range [3], [14]. Thus, there is a growing interest to integrate of electrothermal position sensors in emerging MEMS devices and to understand their physics and dynamic behavior [15]-[22].

Manuscript received March 30, 2014; revised May 18, 2014; accepted May 19, 2014. Date of publication May 23, 2014; date of current version July 25, 2014. This work was supported in part by the Australian Research Council and in part by the University of Newcastle, Callaghan, NSW, Australia. The associate editor coordinating the review of this paper and approving it for publication was Dr. E. H. Yang.

The authors are with the School of Electrical Engineering and Computer Science, University of Newcastle, Callaghan, NSW 2308, Australia (e-mail: ali.bazaei@newcastle.edu.au; reza.moheimani@newcastle.edu.au).

Color versions of one or more of the figures in this paper are available online at http://ieeexplore.iee.org.

Digital Object Identifier 10.1109/JSEN.2014.2326685
Electrothermal displacement sensing was originally used in a silicon accelerometer [23]. Binnig et al. [1] used this concept in an atomic force microscope (AFM) cantilever. However, the electrothermal displacement sensing scheme in [1] suffers from nonlinearity and limited dynamic range [3]. The dynamic range, linearity, and drift of electrothermal sensors can significantly be improved by fabricating two resistors that operate differentially, as noted in [3], [24]-[26]. In [19], [27], and [28] similar electrothermal position sensors were fabricated using a single-layer MEMS fabricarion process.

In [25], electrothermal position sensors were analyzed using a system approach. The effect of nonuniform temperature distribution along the heaters was not considered in this analysis. This effect was considered for heaters with uniform width and dopant concentration using first principles in [29] and through a software-based lumped capacitance model in [19]. The nonuniform heat distribution causes the central area of the sensing resistor to reach temperatures much higher than the outer areas ([20, Fig. 8]). By appropriately shaping the heater width profile for a more uniform temperature distribution, the sensitivity, noise, and linearity of the sensor can be improved [30]. An important step before fabrication is to predict the sensor response by reliable models capturing the various nonlinearities involved [31]. In contrast to commercial modeling software, first-principles-based models provide more transparent, flexible, and reliable tools for analysis, especially when the nonlinear phenomena are dominant.

In this paper we develop analytical models for electrothermal position sensors. The models include and explain a variety of nonlinear phenomena and predict the sensor output with high accuracy. The model is also used to assess the displacement sensor linearity. This measure is experimentally hard to characterize, especially for highly linear MEMS position sensors, due to interfering effects such as noise and limited resolution of micro-system analyzers and also nonlinearities in displacement source such as quadratic dependency of force on actuator voltage in comb drives [32]. The model can also predict the sensor response for much larger displacements than those achievable by limited stroke comb drives in [14] and [30]. Compared to the previously reported models, the proposed first-principles-based model incorporates the following nonlinear phenomena in the heat equation:

- Non-uniform distribution of the temperature.

- Nonlinear temperature profile of resistivity.

- Non-uniform beam width of the shaped heaters.

- Different cross sectional areas for thermal and electrical 


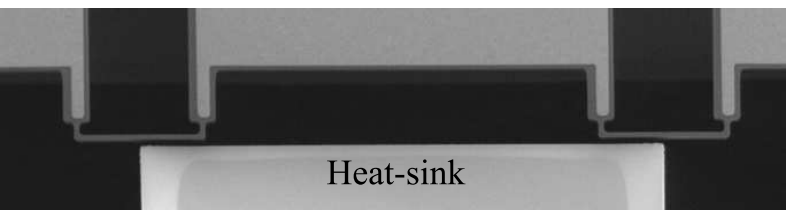

(a)

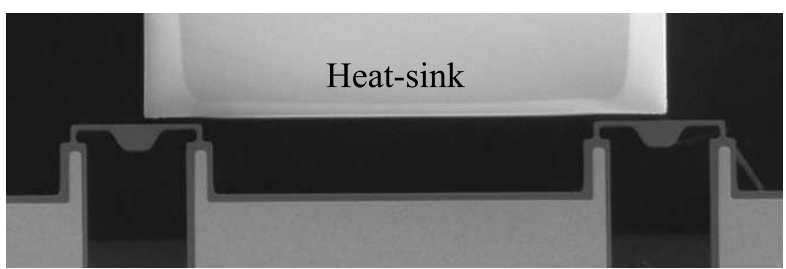

(b)

Fig. 1. SEM images of electrothermal position sensors with heater length of 50 micron [30]. (a) Sensor with straight heaters. (b) Sensor with shaped heaters.

conductions to account for the non-uniform dopant depth profile.

- Nonuniform and position-dependent per-unit-length heat conduction to ambient due to the non-uniform proximity of the heat sink.

- Model complexities arising from biasing heaters with a voltage source rather than a current source, rendering a time-varying nonlinear partial differential heat equation into an integro-differential equation.

In contrast to the first-principles approach in [29], the proposed model considers the non-uniform distribution of electric power along the heater, enabling large signal analysis of the problem with a pre-specified heater current or voltage. Compared to the sophisticated software used in [19], the proposed approach allows using conventional numerical solvers such as MATLAB with desired accuracy and flexibility.

The paper is organized as follows. In Sec. II, a static analysis of the electrothermal position sensor is proposed, considering the effects of both straight and shaped heaters on the sensitivity and linearity of the sensor in constant bias voltage. In Sec. III, we propose an approach based on a parabolic-elliptic partial differential-equation to solve the transient response of the sensor to a step change in heater current. If the heater is excited by a step voltage, the transient problem becomes a timevarying nonlinear partial integro-differential equation, which can be solved by the iterative method proposed in Sec. III-A. Sec. III-B demonstrates the experimental results confirming the proposed transient analyzes. In Sec. III-C, sensor responses to a step displacement change are considered in both constant voltage $(\mathrm{CV})$ and constant current $(\mathrm{CC})$ bias modes. Sec. IV evaluates the sensing bandwidth and linearity of the sensor by imposing pure sinusoidal excitation of the displacement for both straight and shaped heaters in both $\mathrm{CC}$ and $\mathrm{CV}$ bias modes. The validity of the proposed approaches are consistently examined by comparing the simulation results with the experimental data.

\section{StatiC ANALYSis}

Fig. 1 displays the SEM images of two fabricated differential electrothermal sensors using straight and shaped beams

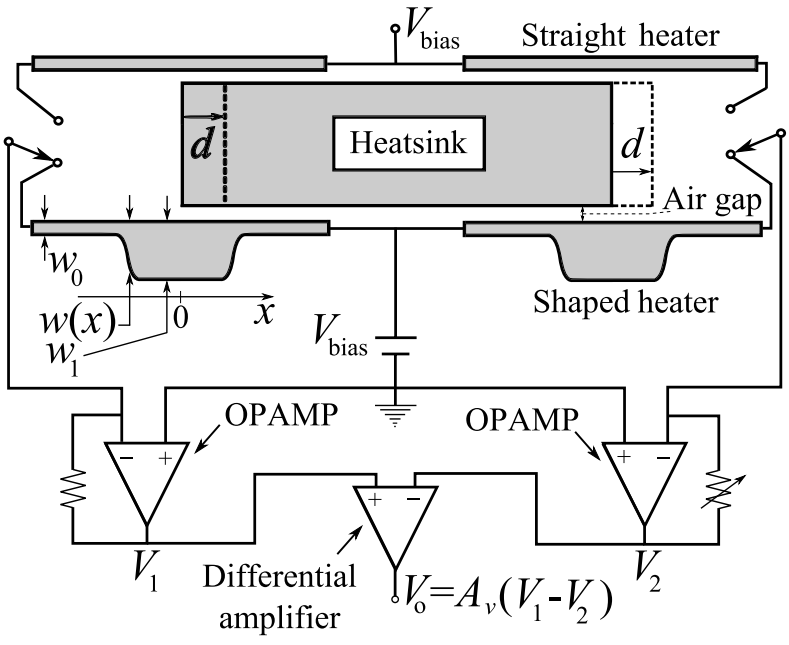

Fig. 2. Circuit diagram and schematic of sensor heaters with beam width profile $w(x)=b+a \tanh \left[\alpha\left(x_{0}-|x|\right)\right]$ and constant bias voltage.

TABLE I

PARAMETER VALUES FOR $50 \mu m$ BEAM HEATERS

\begin{tabular}{ccccccc}
\hline Parameters & $\begin{array}{c}w_{0} \\
(\mu m)\end{array}$ & $\begin{array}{c}w_{1} \\
(\mu m)\end{array}$ & $\begin{array}{c}a \\
(\mu m)\end{array}$ & $\begin{array}{c}b \\
(\mu m)\end{array}$ & $\begin{array}{c}\alpha \\
(\mu m)-1\end{array}$ & $\begin{array}{c}x_{0} \\
(\mu m)\end{array}$ \\
\hline Shaped & 2 & 10 & 4 & 6 & 1 & 7.5 \\
\hline Straight & 2 & 2 & 0 & 2 & N/A & N/A \\
\hline
\end{tabular}

to measure the in-plane position of a heat-sink. Electrostatic actuators are used to move the heat-sink and comb drives connected to it are electrically grounded. This configuration and the structural separation of the heaters and the heatsink by air gaps minimize the crosstalk between the actuator and sensor. The schematic in Fig. 2 simultaneously shows two position sensors, each using a pair of identical heaters, one sensor with uniform beam width (straight) and the other one with nonuniform width (shaped), as described in [30]. To measure the displacement $d$ of the heat-sink, which results in a variation in the individual heat conductances between the heaters and ambient in opposite directions, the resulting small difference between the resistances of the heaters is electronically amplified as the output voltage $V_{o}$. The parameters of the beam width profile $w(x)$, as defined in Fig. 2, have the values reported in Table I, where $x$ is the longitudinal beam position of the heater, $x_{0}$ is the position of maximum slope, and $\alpha$ is a scaling factor to determine the maximum slope. Parameters $a$ and $b$ are determined from minimum and maximum widths $w_{0}$ and $w_{1}$ as:

$$
\left[\begin{array}{l}
b \\
a
\end{array}\right]=\left[\begin{array}{rr}
1 & \tanh \left[\alpha\left(x_{0}-L / 2\right)\right] \\
1 & \tanh \left(\alpha x_{0}\right)
\end{array}\right]^{-1}\left[\begin{array}{l}
w_{0} \\
w_{1}
\end{array}\right],
$$

where $L$ is the beam length.

To roughly estimate the temperature profile of resistivity, we first neglect the minor effect of the heat-sink on the heater resistance and use the following static boundary value problem reported in [30] to predict the I-V characteristic of 
(a)

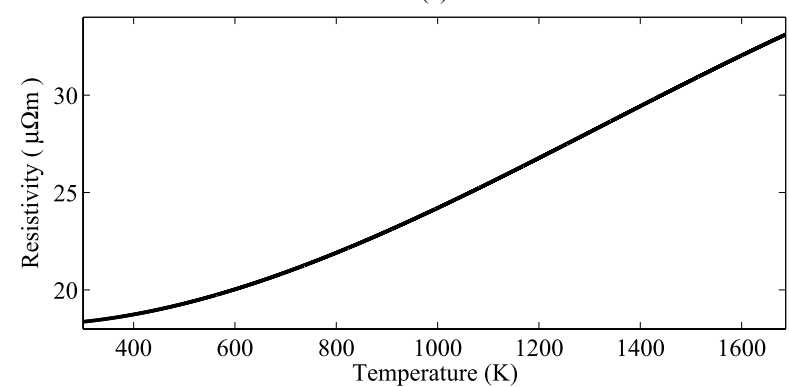

(b) For one heater with length $50 \mu \mathrm{m}$

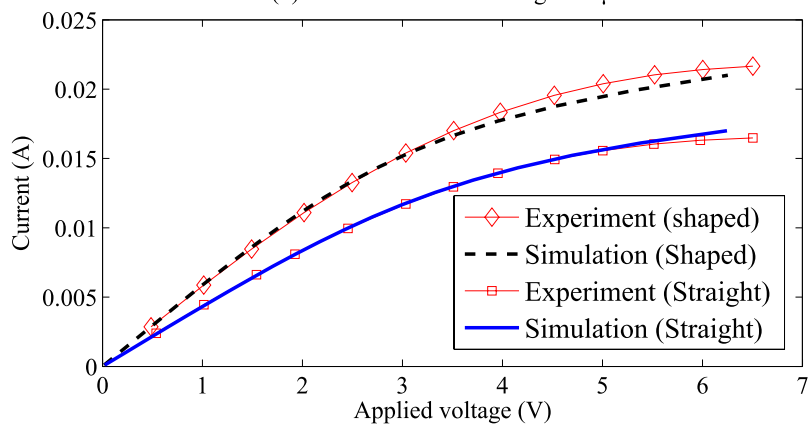

Fig. 3. (a) Resistivity profile. (b) I-V characteristics of the heaters.

the heaters.

$$
\begin{gathered}
\frac{I^{2}}{A_{e}(x)} \rho_{e}(\widetilde{T})+k \frac{d}{d x}\left[A(x) \frac{d \widetilde{T}}{d x}\right]=K_{a}(x) \widetilde{T}(x) \\
{\left.\left[G \widetilde{T}(x) \pm k A(x) \frac{d \widetilde{T}}{d x}\right]\right|_{x= \pm \frac{L}{2}}=0}
\end{gathered}
$$

Here, $\widetilde{T}(x)$ is the local temperature increase of the heater compared to the ambient temperature, $\rho_{e}(\widetilde{T})$ is the temperature dependent resistivity, $A_{e}(x)=(2 \mu m) \times w(x)$ is the local cross-sectional area through which electric current $I$ flows, $k=130 \frac{\mathrm{W}}{K m}$ is the heater thermal conductivity, $A(x)=$ $(25 \mu \mathrm{m}) \times w(x)$ is the local cross-section of the heater, $G$ is the heat conductance to ambient from each end point of the heater connected to a pad, and $K_{a}(x)$ is the local per-unitlength thermal conductance of the heater to ambient, which in the absence of the heat-sink is described as [30]:

$$
K_{a}(x)=0.03 \times \frac{w(x)+25 \mu \mathrm{m}}{27 \mu \mathrm{m}}, \quad\left(\frac{\mathrm{W}}{\mathrm{Km}}\right)
$$

We started with $G=70 \frac{\mu W}{K}$ and the bell-shaped temperature profile of resistivity in [30], which is theoretically predicted for a uniform doping concentration [29]. The I-V characteristics of both straight and shaped heaters were predicted and compared with the experimental data. To lower the prediction errors for both heaters, the heat conductance $G$ and the resistivity profile were then changed several times and the updated heat equations were solved each time. For the subsequent analysis, we opted $G=50 \frac{\mu W}{K}$ and the resistivity profile shown in Fig. 3(a), which is adequately approximated by a third order polynomial. This profile yields a reasonable match with the experimental data for both straight and shaped beams, as shown in Fig. 3(b). Note that the bell-shaped resistivity profile in [30] cannot predict the experimental I-V data of both

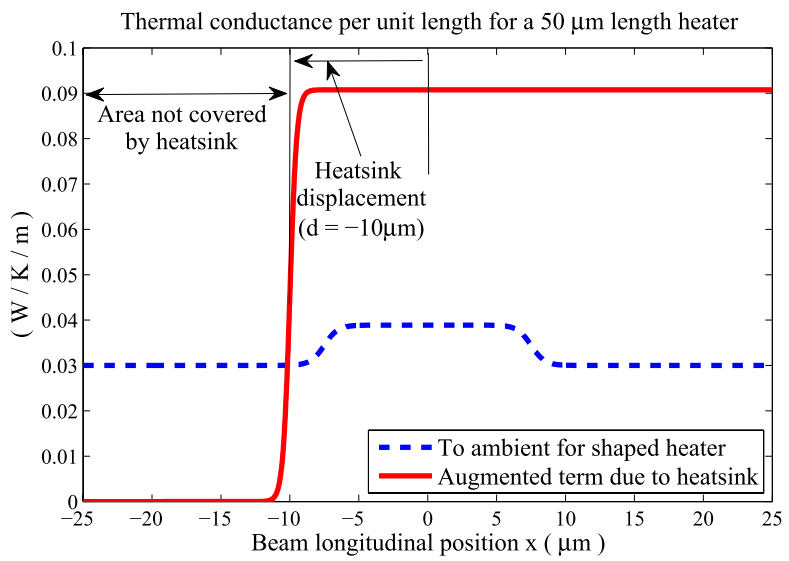

Fig. 4. Two terms of per-unit-length thermal conductance between the heater and ambient. The dashed line is the first term without the heat-sink effect described by Eq. (4). The solid line is the second term due to the heat-sink described by Eq. (5).

heaters, especially at high temperatures due to large variations in the dopant concentration along the heater's depth.

To consider the effect of the heat-sink, the per-unit-length thermal conductance $K_{a}$ between the heater and ambient environment is partially augmented based on the heat-sink displacement $d$, as illustrated in Fig. 4, using the following additional term.

$$
\Delta K_{a}(x, d)=0.0454\left[1+\tanh \left(\frac{x \mp d}{0.5 \mu \mathrm{m}}\right)\right], \quad\left(\frac{\mathrm{W}}{\mathrm{Km}}\right)
$$

where the minus and plus signs before $d$ correspond to the left and right heaters in Fig. 2, respectively.

Assuming the device was fabricated flawlessly, the resistances of the left and right heaters, denoted by $R_{1}(d)$ and $R_{2}(d)$, are equal when the displacement is zero. Hence, with an identical value of $R_{f}$ for the feedback resistances in Fig. 2, the sensor generates a zero output voltage at zero displacement. This approximation allows us to predict the sensor output voltage as follows:

$$
V_{o}(d)=A_{v} V_{\text {bias }} R_{f}\left[\frac{1}{R_{2}(d)}-\frac{1}{R_{1}(d)}\right]
$$

Fig. 5 shows the predicted sensor outputs when $A_{v}=90$, $R_{f}=330 \Omega$, and both sensors use similar circuits and bias voltages $(6.1 \mathrm{~V})$ as in [30]. The close agreement of these results with experimental data, illustrated in Fig. 5 inset, further confirms the validity of the proposed model. As indicated by the variable $\delta$ in Fig. 5, the static characteristics of the shaped sensor is about four times closer to a perfect linear response than the straight sensor. Since with an equal bias voltage the shaped sensor is warmer than the straight sensor, we also reduced the bias voltage of the shaped sensor to $5.24 \mathrm{~V}$ so that its average temperature becomes equal to that of the straight sensor with $6.1 \mathrm{~V}$ bias, as shown in Fig. 6. Fig. 5 also includes simulation corresponding to this case, where a further improvement in linearity is achieved plus an increased sensitivity compared to the straight sensor.

Remark 1: In practice, exposure to air in room temperature and formation of a thin Silicon dioxide layer on the heater 


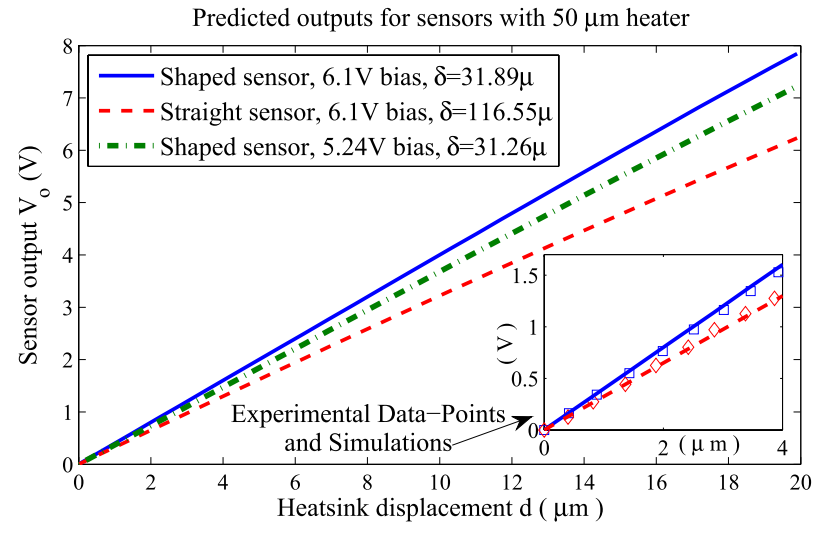

Fig. 5. Comparison of sensor output versus stage displacement for curved and straight sensors. $\delta=1-\left|\rho_{d, V_{o}}\right|$ is a measure of deviation from linearity, where $\rho_{d, V_{o}}$ is Pearson correlation coefficient, whose maximum absolute value is 1 for a perfectly linear response [33]. The inset is a close-up of the results along with limited range measured data from [30, Fig. 9].

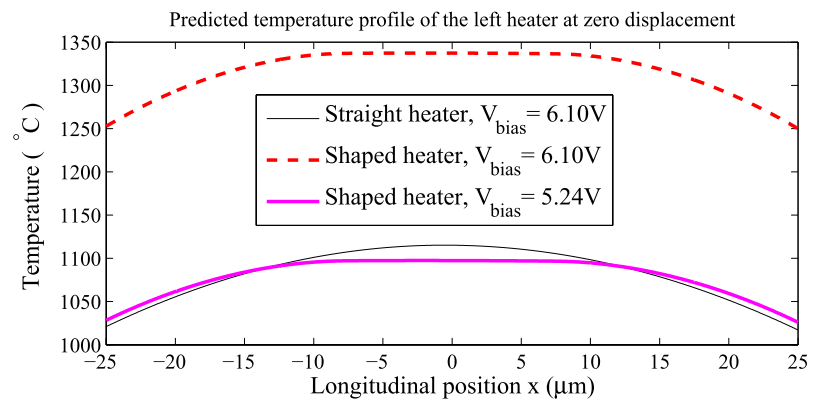

Fig. 6. Comparison of the predicted temperature profile of the left straight heater with that of the shaped heater at zero displacement of the heat-sink and under two different conditions: similar bias voltages and similar average temperatures.

surface stop further thermal oxidation of the beam [34], enabling the heater to tolerate the high temperatures predicted in Fig. 6.

Remark 2: For thermal conductance $G$ at the heater endpoints and the overall per-unit-length thermal conductance $K_{a}(x, t)=K_{a}(x)+\Delta K_{a}(x, d(t))$, we opted simple forms with the parameters selected consistent with the experimental data. These profiles and parameters can hardly be determined based on first principles, as the analysis usually requires solving a heat equation in two or three dimensions and nonlinear approximation of shape factors [35].

Remark 3: With the increase of heater temperature, the air gap between the heater and heat-sink may nonuniformly decrease due to thermal expansion of the beam and buckling. This effect can change the profile of the augmented per-unitlength heat conductance $\Delta K_{a}(x, d)$, to some extent. However, the reduction of gap is negligible for a heater with long legs, where their compliance prevents buckling of the sensing part [19]. For Silicon heaters with short legs, 2 microns width, and sensing lengths less than 100 microns, observations at very high temperatures also confirm a negligible gap reduction [20], [30].

Remark 4: In the fabricated sensors considered for experimental validations, profile of dopant concentration is not precisely available. However, during the fabrication, a lightly doped Silicon wafer with a minimum resistivity of $10^{4} \mu \Omega \mathrm{m}$ is heavily doped through one hour annealing at $1050^{\circ} \mathrm{C}$ in Argon with a phosphosilicate glass (PSG) layer deposited on the top surface [36]. This is an initial standard process to establish ohmic contact with Silicon for subsequent metal pads. Although, the thickness of device layer is 25 microns, we assume a narrow width of 2 microns for the heavily doped zone, which affected the electrical cross-section $A_{e}(x)$ of the heater. The 2-micron-depth is also consistent with the experimental dopant profiles reported in [37]. As shown in Fig. 3 (a), the average predicted resistivity in the heavily doped zone is at least 300 times lower than that of the originally lightly doped wafer. Hence, it is mainly the heavily doped zone that determines the heater resistance.

\section{TRANSIENT ANALYSIS}

To model the sensor dynamics, we consider the heat equation governing a single heater reported in [30], which can be rearranged in the form of a parabolic-elliptic partialdifferential-equation in one dimension as:

$$
C(x) \frac{\partial \widetilde{T}(x, t)}{\partial t}=\frac{\partial}{\partial x} F\left(x, \frac{\partial \widetilde{T}}{\partial x}\right)+S\left(x, t, \widetilde{T}, \frac{\partial \widetilde{T}}{\partial x}\right),
$$

where functions $C(x), F\left(x, \frac{\partial \widetilde{T}}{\partial x}\right)$, and $S\left(x, t, \widetilde{T}, \frac{\partial \widetilde{T}}{\partial x}\right)$ are defined as:

$$
\begin{aligned}
C(x) & :=\operatorname{c\rho A}(x) \\
F\left(x, \frac{\partial \widetilde{T}}{\partial x}\right) & :=k A(x) \frac{\partial \widetilde{T}}{\partial x} \\
S\left(x, t, \widetilde{T}, \frac{\partial \widetilde{T}}{\partial x}\right) & :=\frac{I^{2}(t) \rho_{e}(\widetilde{T})}{A_{e}(x)}-K_{a}(x, t) \widetilde{T}(x),
\end{aligned}
$$

where constants $c=712 \frac{\mathrm{J}}{\mathrm{kg} \cdot \mathrm{K}}$ and $\rho=2329 \frac{\mathrm{kg}}{\mathrm{m}^{3}}$ refer to specific heat capacity and density of the heater material, respectively. We can also write the boundary conditions (3) in the following from:

$$
\left.\left[P^{\mp}(\widetilde{T}(x))+Q^{\mp} F\left(x, \frac{\partial \widetilde{T}}{\partial x}\right)\right]\right|_{x=\mp \frac{L}{2}}=0,
$$

where functions $P^{\mp}(\widetilde{T})$ and $Q^{\mp}$ are defined as:

$$
P^{-}(\widetilde{T})=P^{+}(\widetilde{T})=G \widetilde{T}(x) ; \quad Q^{+}=-Q^{-}=1 .
$$

Although the readout circuit in Fig. 2 corresponds to a voltage source applied to the heaters, it can be easily converted to an equivalent current source if the position of each heater is swapped with the corresponding feedback resistance, as shown in Fig. 7. In this case, a similar differential sensing scheme requires fabricated heaters with no common pads. Assuming a known electric current $I(t)$, accurate solution of the above distributed-parameter nonlinear dynamic equation can be obtained by standard solvers such as pdepe in MATLAB. For example, if the left shaped heater is excited at $t=0$ by a constant current source of $19.87 \mathrm{~mA}$, which generates a voltage of $5.24 \mathrm{~V}$ across the heater in steady-state, we obtain the temperature profiles shown in Fig. 8 during the transient. The time history of the heater resistance during this transient 


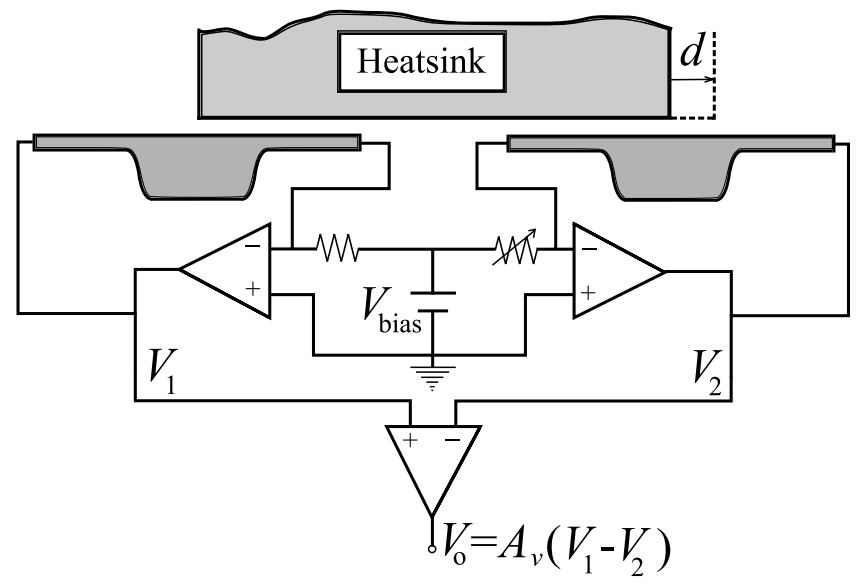

Fig. 7. Differential sensor circuit in constant bias current mode.

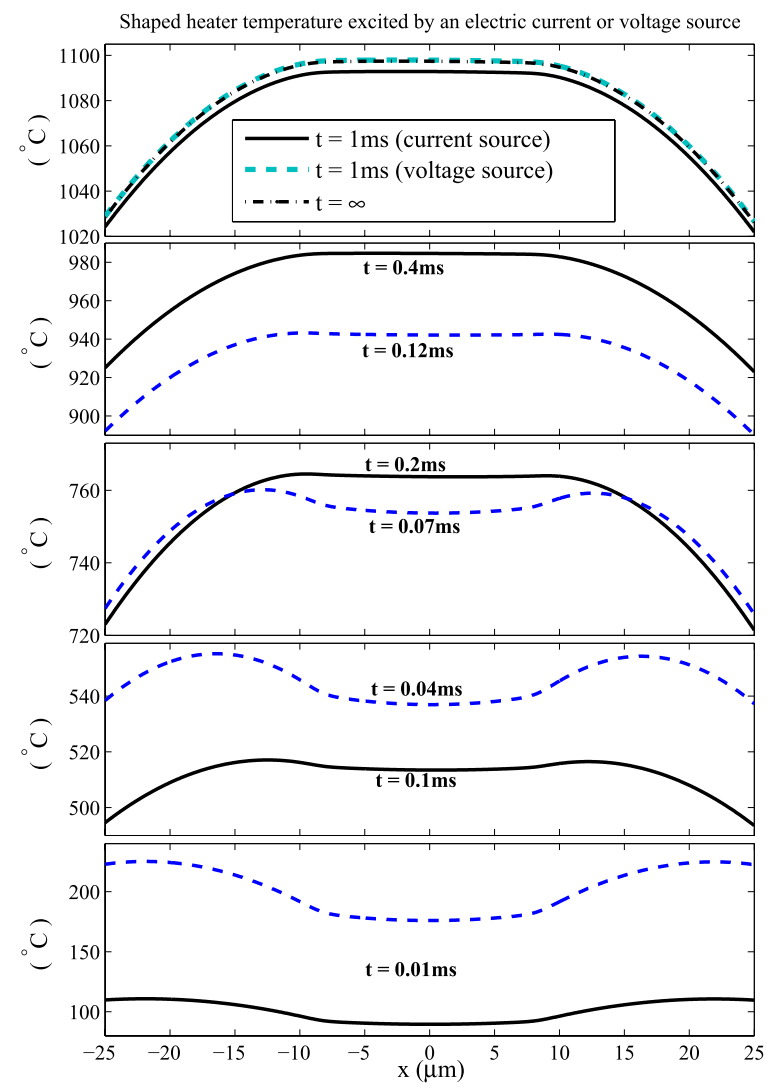

Fig. 8. Temperature profiles of the left heater at zero displacement of the heat-sink and room temperature initial condition $\left(27^{\circ} \mathrm{C}\right)$, during a transient excited by a step current source of $19.87 \mathrm{~mA}$ (solid curves) and also by a step voltage source of $5.24 \mathrm{~V}$ (thick dashed lines).

response, depicted in Fig. 9, has a time constant of $0.22 \mathrm{msec}$, indicating a bandwidth of $727 \mathrm{~Hz}$ when exciting the shaped heater with a current source.

\section{A. Voltage Driven Sensor}

The problem is more complicated if exciting the heaters with a voltage source $V_{\text {bias }}$, as depicted in Fig. 2. In this case, the time history of the electric current $I(t)$ is not known in advance but is related to the time-varying heater resistance

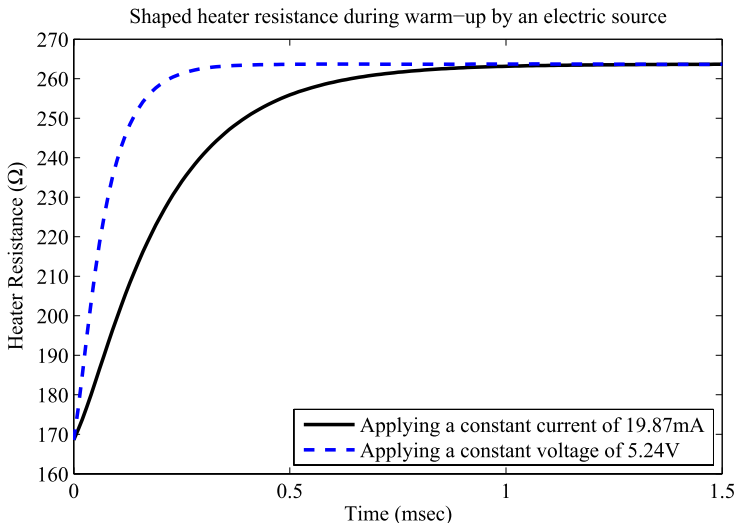

Fig. 9. Time histories of the left shaped heater at zero displacement of the heat-sink and room temperature initial condition $\left(27^{\circ} \mathrm{C}\right)$, during a transient excited by a step current source of $19.87 \mathrm{~mA}$ (solid curve) and also by a step voltage source of $5.24 \mathrm{~V}$ (dashed curve).

$R_{h}(t)$ as:

$$
\begin{aligned}
R_{h}(t) & =\int_{-\frac{L}{2}}^{\frac{L}{2}} \frac{\rho_{e}[\widetilde{T}(x, t)]}{A_{e}(x)} d x \\
I(t) & =\frac{V_{\text {bias }}}{R_{h}(t)}
\end{aligned}
$$

This leads to a nonlinear partial integro-differential equation that cannot be numerically solved by a standard MATLAB solver. However, we were able to solve this problem using the same solver when the heater was driven by a step voltage of $5.24 \mathrm{~V}$, through iterations. We started from the solution to a step current source of $I_{0}(t)=19.87 \mathrm{~mA}$ for $t>0$, whose initial and steady-state solutions are equal to those of the constant voltage source of $5.24 \mathrm{~V}$ and has the heater resistance profile shown in Fig. 9 (solid line), denoted by $R_{h_{0}}(t)$ in the iteration. Assuming that the time histories of the heater resistance are the same in both problems, the current required to maintain the constant voltage of $5.24 \mathrm{~V}$ may be updated as $\frac{5.24 V}{R_{h_{0}}(t)}$, which is not identically constant. Using this current profile, we may solve the transient problem by the method proposed in Sec. III and obtain an updated resistance time profile $R_{h_{1}}(t)$ for the heater to update the current profile for the next iteration as $\frac{5.24 V}{R_{h_{1}}(t)}$. Although, iteratively updating the current in this way leads to a divergent solution, we found that selecting the updated current profile $I_{i+1}(t)$ as a linear combination of $\frac{5.24 V}{R_{h_{i}}(t)}$ and $I_{i}(t)$, i.e.:

$$
I_{i+1}(t)=\beta I_{i}(t)+(1-\beta) \frac{5.24 V}{R_{h_{i}}(t)}, \quad i=0,1,2, \ldots,
$$

where $\beta$ is a constant, can lead to a convergent solution for the constant voltage problem. Results of only ten iterations, shown in Fig. 10 for $\beta=0.5$, demonstrate a rapid convergence of the proposed algorithm to the constant voltage source problem. The resulting transient solutions for the constant voltage source problem, depicted in Figures 8 and 9, indicate a time constant of $0.077 \mathrm{~ms}$ for the increase of the heater resistance. Hence the warm-up bandwidth of the heater when excited by a voltage source is about $2 \mathrm{kHz}$, which is almost 3 times faster than that of the current source excitation. 

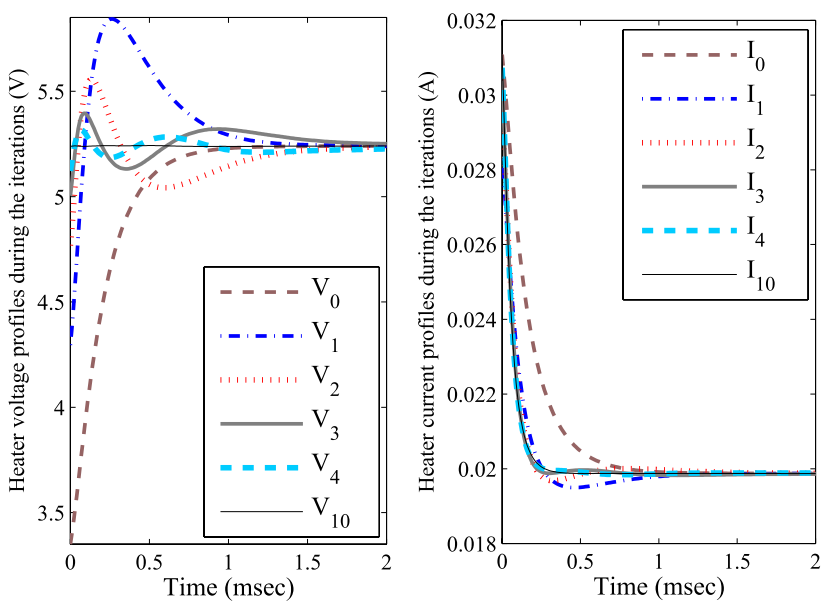

Fig. 10. Time histories of the left shaped heater at zero displacement of the heat-sink and room temperature initial condition $\left(27^{\circ} \mathrm{C}\right)$, when iteratively updating the current profile according to (15) to obtain a solution when the heater is excited by a constant voltage source of $5.24 \mathrm{~V}$ at $t=0$.

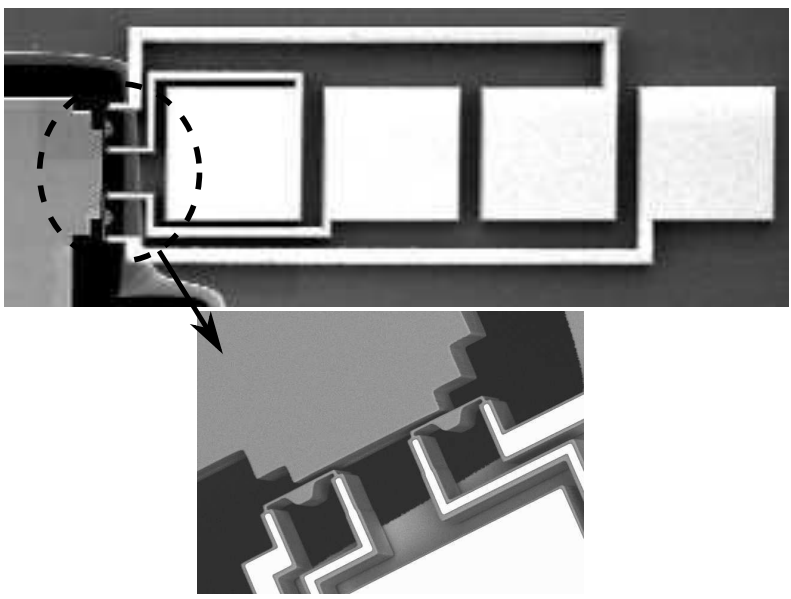

Fig. 11. SEM images of the electrothermal position sensor with $50 \mu \mathrm{m}$ shaped heaters and no common pads [38], [39].

\section{B. Experimental Results}

To validate the above analysis we performed a number of experiments on a MEMS device with a pair of similar shaped heaters with no common pads. Fig. 11 shows the SEM images of the fabricated device [38], [39]. This arrangement enables us to test validity of our analysis of the sensors when they are operated in the constant current mode. Fig. 12 demonstrates the experimental step responses of the sensor for both constant voltage and constant current modes, which have been normalized for comparison purposes. To ensure similar thermal conditions for the heaters in both modes, the bias voltage in the constant current mode was set to yield the same steadystate voltage across the heater as in the constant voltage mode. Note that the experimental results are qualitatively consistent with the simulations in Fig. 9, confirming the validity of the model and more achievable sensor bandwidth by applying a constant bias voltage to the heater.

\section{Response to Displacement Change}

Assuming zero displacement, we have already simulated and experimentally tested the transient response of the heater

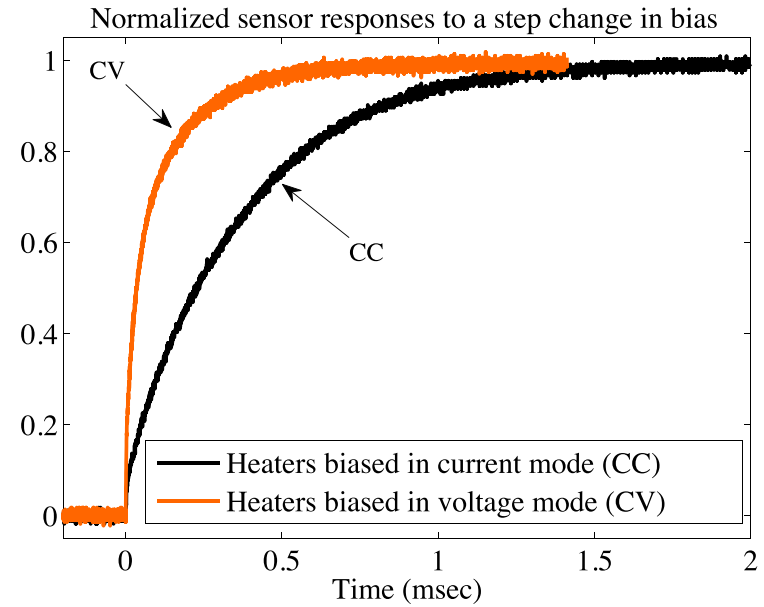

Fig. 12. Experimental step-responses of the sensor, when shaped heaters are biased by voltage and current sources. In each bias mode, the source was tuned to maintain 5 volts on the heaters so that heaters have almost the same temperature in both cases. Then, a small square wave signal was added to the source and its amplitude was tuned so that the heater voltage varies about $200 \mathrm{mV}$ peak-to-peak in each case.

to a step change in the electrical bias of the sensor. Although the limited mechanical bandwidth and actuation limits make it impossible to realize ideal step discontinuities in the displacement, the proposed simulation strategy can predict the sensor transient response to such changes, while including the nonlinearities.

Fig. 13, shows the response of the left $50 \mu m$-length shaped heater to a $10 \mu \mathrm{m}$ step change in the heat-sink position. In constant current and voltage modes, we maintain the heater current and voltage equal to $19.87 \mathrm{~mA}$ and $5.24 \mathrm{~V}$, respectively. Both modes have identical temperature profiles as shown in Fig. 6. This is due to their initial conditions that correspond to a zero displacement. As indicated earlier, it is more convenient to solve the time-varying heat equation (7) first in constant current mode with a nonzero displacement $d=10 \mu \mathrm{m}$, which affects the system through (5) and (10). For the constant voltage mode, we start from the constant current mode solution and use the iterative method described in Sec. III-A. The voltage mode results presented in Fig. 13 correspond to the tenth iteration, where the updated current profile yields an almost constant voltage of $5.24 \mathrm{~V}$ on the heater during the transience. The settling times corresponding to the variations of the normalized heater resistances in Fig. 13(b), which are caused by the step displacement, are almost similar to the response times in Fig. 10 obtained by step bias changes. These simulations further verify that the speed of position sensing can be measured through the bias voltage in electrothermal position sensors [3], [30].

The steady-state resistance changes resulting from the 10 micron displacement in the constant voltage and current modes, denoted by $\Delta R_{\max }$ in Fig. 13(b), are $0.828 \Omega$ and $2.424 \Omega$, respectively, indicating a higher static sensitivity for the current mode. This result relatively agrees with the sensitivities reported in [19] for voltage and current modes and further validates the proposed first-principles-based modeling approach. 
(a)
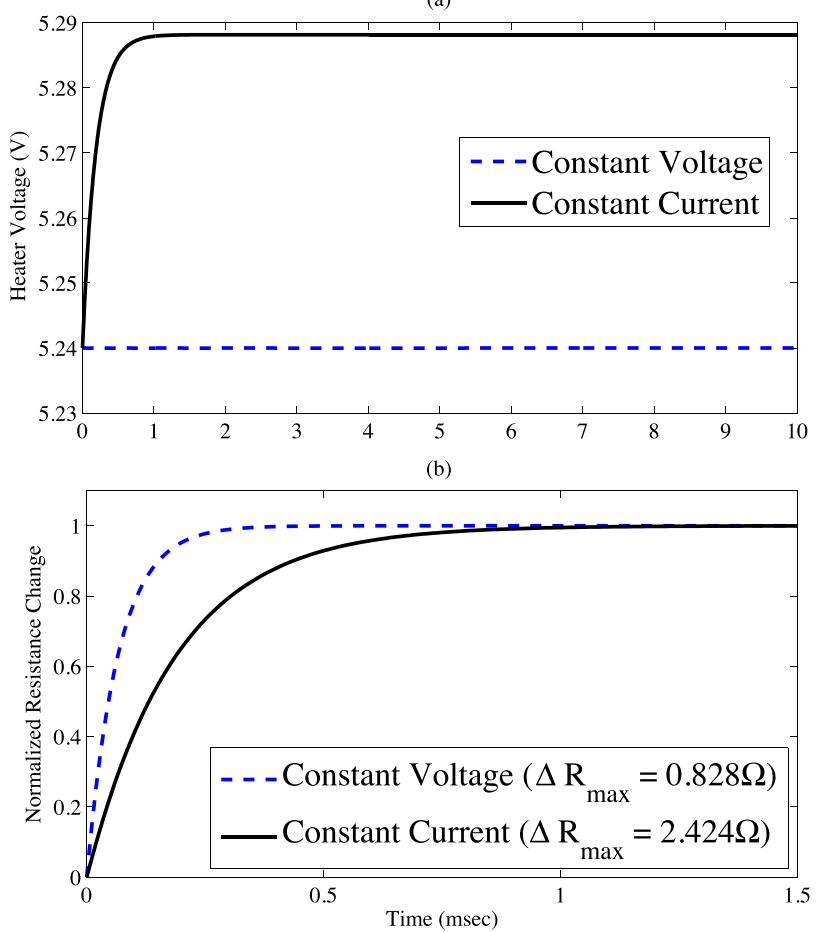

Fig. 13. Simulated transient responses to a $10 \mu \mathrm{m}$ step change in the heatsink position at $t=0$, when the left $50 \mu \mathrm{m}$-length shaped heater is biased by a voltage or a current source. In each bias mode, the initial conditions are set to the steady-state solution of temperature at zero heat-sink displacement, while a constant heater voltage of $5.24 \mathrm{~V}$ or a constant current of 19.87 $\mathrm{mA}$ is applied. The change in each heater resistance has been divided by its final value (denoted by $\Delta R_{\max }$ ) for better comparison of both bias modes (a) Heater voltage after a step displacement. (b) Change of heater resistance after a step displacement.

\section{SENSING BANDWIDTH AND LINEARITY}

Using the proposed model, we investigate the sensing bandwidth and linearity of the electrothermal sensor for both straight and shaped heaters under current and voltage bias modes. We impose an exact sinusoidal motion on the heat-sink displacement $d(t)$, which affects the system through Eqs. (5) and (10), and solve for both heaters responses. Then, the sensor outputs in constant voltage and current modes are calculated by Eq. (6) and the following relationship, respectively:

$$
V_{o}^{c c}=A_{v} \frac{V_{\text {bias }}}{R_{i}}\left[R_{2}(d)-R_{1}(d)\right],
$$

where $R_{i}$ refers to the input resistances of the trans-impedance amplifiers in the constant current mode readout circuit, which are assumed identical. Note that driving the heat-sink position to follow an exact sinusoidal trajectory is almost impossible in practice due to actuation nonlinearity and sensing inaccuracies, justifying the model-based analysis. Before running each sine wave test, we apply a constant step displacement with the same amplitude of the intended sinusoid to find the constant factor that converts the output voltage to displacement. The conversion factor is then used to generate the displacement predicted by the sensor during the sine wave test. As in Sec. III-A, the constant voltage $(\mathrm{CV})$ mode problem is iteratively solved using the solution of constant current (CC) mode problem. Fig. 14 shows the responses of the sensor with shaped heaters to

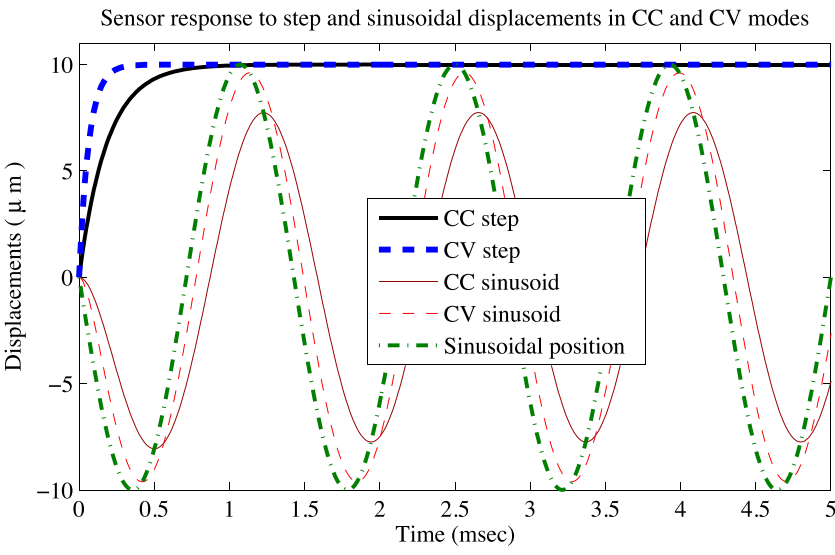

Fig. 14. Simulated transient and steady-state responses of the sensor with shaped heaters to step and $700 \mathrm{~Hz}$ sinusoidal displacements with $10 \mu \mathrm{m}$ amplitudes. Effects of both voltage and current bias modes are demonstrated, where the $50 \mu \mathrm{m}$-length heaters are biased by a $5.24 \mathrm{~V}$ voltage source $(\mathrm{CV})$ or a $19.87 \mathrm{~mA}$ current source (CC), respectively.

step and sinusoidal displacements with 10 micron amplitudes. To approximate the sensor bandwidth from the sinusoidal response, we assume a first-order linear low-pass filter for the sensor dynamics, which yields the following relationships for the cutoff frequency $f_{c}$ :

$$
\begin{aligned}
& f_{c}=\frac{f}{\tan (\phi)} \\
& f_{c}=\frac{f}{\sqrt{\frac{A_{o}^{2}}{A_{m}^{2}}-1}},
\end{aligned}
$$

where $f$ and $A_{o}$ refer to the frequency and amplitude of the sinusoidal displacement, $A_{m}$ is the amplitude measured by the sensor, and $\phi$ is the phase delay of the measured signal with respect to the actual displacement. Using Eq. (17) and the steady-state component of the $700 \mathrm{~Hz}$ sinusoidal responses in Fig. 14, the sensor with shaped heaters has bandwidths $862 \mathrm{~Hz}$ and $2380 \mathrm{~Hz}$ in $\mathrm{CC}$ and $\mathrm{CV}$ modes, respectively. Alternatively, we may estimate each bandwidth by the reciprocal time constant of the step response in Fig. 14, which yields consistent results of $855 \mathrm{~Hz}$ and $2415 \mathrm{~Hz}$ for $\mathrm{CC}$ and $\mathrm{CV}$ modes, respectively.

Fig. 15 shows the voltage and resistance values of the left shaped heater during the step and sinusoidal excitations of the displacement in both CC and CV bias modes. As in Fig. 13 (a), the flat response of the heater voltage in Fig. 15 (a) confirms valid convergence of the iterative method in the constant voltage case. As the displacement is a pure sinusoid, we can solely assess the nonlinearity of the sensor by calculating the amplitudes of the higher-order harmonics in the Fourier series expansion of the steady-state sensor output. The amplitude of each harmonic is divided by the amplitude of the fundamental component in each Fourier series for comparison purposes. The results for the second and third harmonics are presented in Table II, where those associated with one heater resistance are also included. Note that the heater resistance profile, as shown in Fig. 15 (b), has a very large DC term, which should be canceled for accurate computation of the fundamental 
(a)
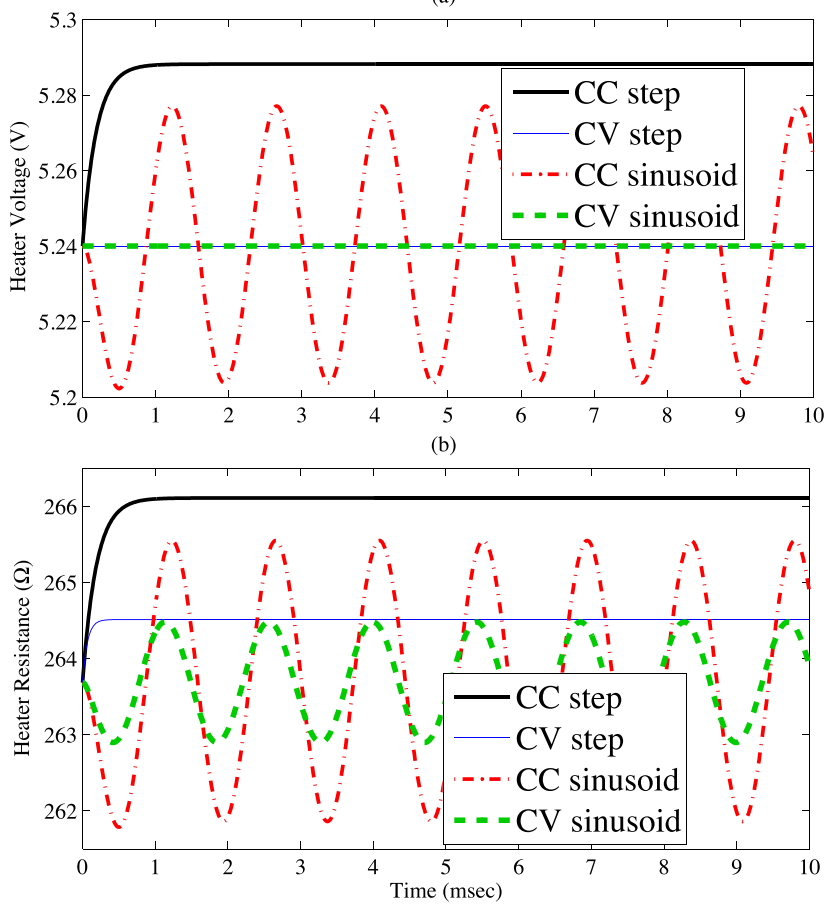

Fig. 15. Voltage and resistance values of the left shaped heater in response to the step and the $700 \mathrm{~Hz}$ sinusoidal displacements with 10 micron amplitudes (corresponding to Fig. 14). (a) Response of left heater voltage to step and sinusoidal displacements. (b) Left heater resistance with step and sinusoidal displacements.

\section{TABLE II}

Percentages of the SeCond And Third Harmonics Compared to Their Fundamental Component In the Responses of the LeFT Heater Resistance $\left(R_{1}\right)$ AND the Differential Sensor OUtPut $\left(V_{o}\right)$ to a Pure Sinusoidal Displacement in CC AND CV Bias Modes, For SENSORS With SHAPEd AND STRAight HEATERS Biased Around Similar Average Operating Temperature (CORRESPONDING TO Figs. 14 AND 15 FOR THE SENSOR USING SHAPED HEATERS AND FIGS. 16 AND 17 FOR SENSOR Using the Straight Heaters)

\begin{tabular}{c|c|c|c|c}
\hline \multicolumn{5}{c}{ Shaped heater sensor } \\
\hline Harmonic No. & \multicolumn{2}{|c|}{$R_{1}$} & \multicolumn{2}{c}{$V_{o}$} \\
\hline & $\mathrm{CC}$ & $\mathrm{CV}$ & $\mathrm{CC}$ & $\mathrm{CV}$ \\
\hline 2nd & $0.46 \%$ & $0.31 \%$ & $0.01 \%$ & $0.01 \%$ \\
\hline 3rd & $0.06 \%$ & $0.08 \%$ & $0.06 \%$ & $0.08 \%$ \\
\hline \hline \multicolumn{6}{c}{ Straight heater sensor } \\
\hline Harmonic No. & \multicolumn{3}{c|}{$R_{1}$} & \multicolumn{2}{c}{$V_{o}$} \\
\hline \multicolumn{6}{|c|}{$\mathrm{CC}$} & $\mathrm{CV}$ & $\mathrm{CC}$ & $\mathrm{CV}$ \\
\hline 2nd & $0.39 \%$ & $0.28 \%$ & $0.02 \%$ & $0.02 \%$ \\
\hline 3rd & $0.10 \%$ & $0.17 \%$ & $0.10 \%$ & $0.17 \%$ \\
\hline
\end{tabular}

component and its harmonics. Comparing the percentages associated with the sensor output $V_{o}$ (which is differentially obtained from both heaters) with those of a single heater resistance $R_{1}$, it is revealed that the differential action significantly reduces the dominant nonlinearity that exists in the second harmonic of the heater resistance without a noticeable change in the third harmonic distortion. This observation, which agrees with the experimental results reported in [26] for the $\mathrm{CV}$ bias mode, further confirms the validity of the proposed

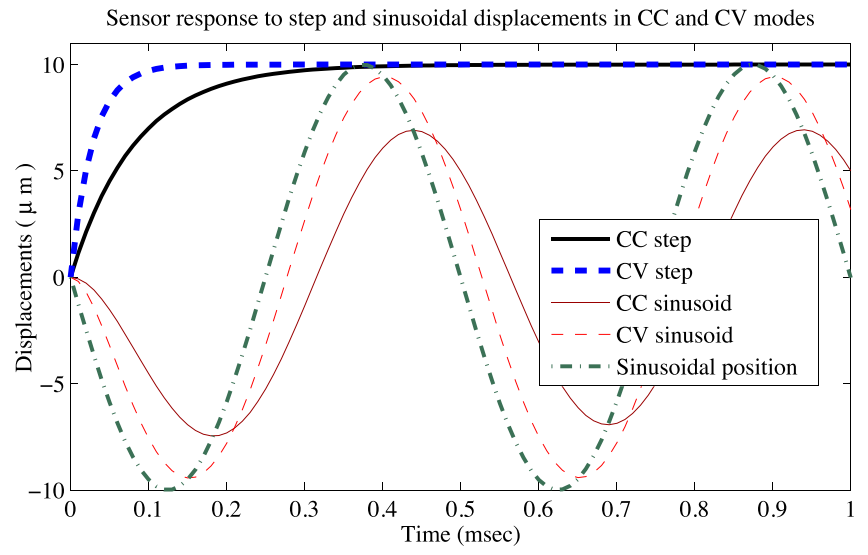

Fig. 16. Simulated transient and steady-state responses of the sensor with straight heaters to step and $2 \mathrm{kHz}$ sinusoidal displacements with $10 \mu \mathrm{m}$ amplitudes. Effects of both voltage and current bias modes are demonstrated, where the $50 \mu \mathrm{m}$-length heaters are biased by a $6.1 \mathrm{~V}$ voltage source $(\mathrm{CV})$ or a $16.92 \mathrm{~mA}$ current source (CC), respectively.
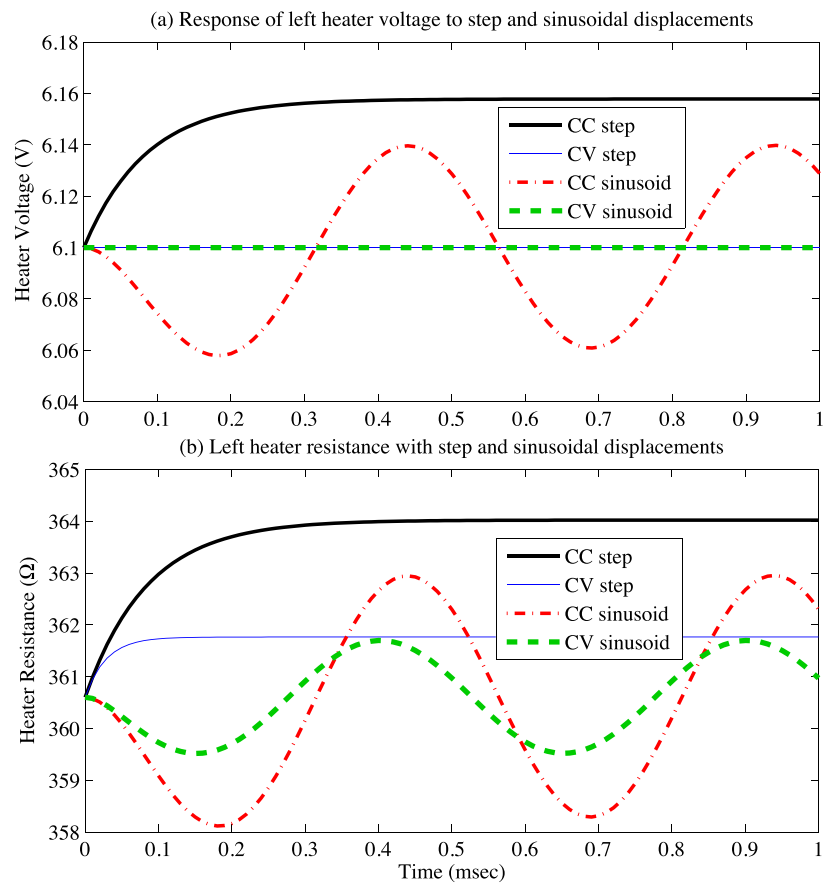

Fig. 17. Voltage and resistance values of the left straight heater in response to the step and the $2000 \mathrm{~Hz}$ sinusoidal displacements with 10 micron amplitudes (corresponding to Fig. 16).

first-principles-based model for differential electrothermal position sensors.

The results presented so far in this section are associated with the shaped heater sensors. For the sake of completeness, we have also performed similar analysis, where straight heaters with parameter and bias values quoted in Sec. II are used in the sensor. Hence, as mentioned at the end of Sec. II, the straight heaters operate at almost the same temperature as the shaped heaters, in order to make a fair comparison. The responses of the sensor with straight heaters to 10-micron-amplitude step and sinusoidal displacements are shown Figures 16, 17, and Table II. As the bandwidth of 
TABLE III

BANDWIDTHS OF THE SENSORS USING SHAPED OR STRAIGHT Heaters in CC or CV Bias Modes, Calculated by Sine WAVE Tests AND EQ. (17) AND (18), OR THE STEP RESPONSES

\begin{tabular}{c|c|c|c|c}
\hline Method & \multicolumn{2}{|c|}{ Shaped sensor } & \multicolumn{2}{c}{ Striaght sensor } \\
\hline & CC & CV & CC & CV \\
\hline Eq. (17) & $0.86 \mathrm{kHz}$ & $2.38 \mathrm{kHz}$ & $1.93 \mathrm{kHz}$ & $5.45 \mathrm{kHz}$ \\
\hline Eq. (18) & $0.86 \mathrm{kHz}$ & $2.40 \mathrm{kHz}$ & $1.93 \mathrm{kHz}$ & $5.66 \mathrm{kHz}$ \\
\hline Step Response & $0.85 \mathrm{kHz}$ & $2.42 \mathrm{kHz}$ & $1.92 \mathrm{kHz}$ & $5.57 \mathrm{kHz}$ \\
\hline
\end{tabular}

the straight heater sensor is higher than the curved one, we increased the sine wave frequency to $2 \mathrm{kHz}$ to accurately compute the phase delay between the displacement excitation and the sensor response. The resulting bandwidths in $\mathrm{CC}$ and CV modes, using Equations (17), (18), or the step responses, are included in Table III, along with those reported before for the curved heater sensor, for comparison purposes. According to this analysis, when switching from shaped heaters to the straight ones, the sensor bandwidth is increases by a factor of 2.3. This theoretical result agrees well with the 2.5 -fold bandwidth increase reported in [30], when performing a similar switching in constant voltage mode, experimentally. The small difference can be due to the fact that here we apply different biases to maintain identical operating temperatures for the heaters, while in [30] identical voltages are applied to the heaters in both cases. As reported in Table III, obtaining close results in each case by the three different methods based on postulating linear first-order model dynamics further justifies the ansatz for approximation of the sensing bandwidth.

A comparison between the harmonic distortions of the sensors using shaped and straight heaters reported in Tables II reveals that the sensor with shaped heaters has a more linear differential output than the sensor with straight heaters in both $\mathrm{CC}$ and $\mathrm{CV}$ modes. These linearity test results, which are obtained from dynamic analysis of the proposed model, are also consistent with the experimental dynamic tests reported in [30] to assess the sensor linearity. Furthermore, in contrast to the sensing bandwidth, the predicted sensor linearity is slightly improved when switching from constant voltage to constant current mode.

\section{CONCLUSION}

Static and dynamic analyzes of differential electrothermal position sensors were reported in this paper. Effects of nonlinearities such as nonuniform temperature profile, position dependent heat-sink proximity, heaters with contoured beam width, and nonlinear temperature dependency of resistivity were included in the analyzes. In both straight and shaped heater cases, the model predicts linear operation of the sensor over a large displacement range and agrees well with the quiescent experimental data. In comparison with uniform width (straight) heaters, the static analysis reveals improved linearity for the sensor using heaters that are shaped for more uniform temperature distribution. The dynamic analyzes were performed with both constant bias current and voltage applied to the heaters, where an iterative procedure was proposed to solve the nonlinear partial integro-differential equation in the bias voltage mode. Compared to the current mode, the model predicts a faster transient response for the sensor, when biased in voltage mode. This fact was experimentally confirmed to validate the model. We also used the model to predict the responses of the sensors to ideal step and sinusoidal variations in displacement, which are impossible tasks to realize. The sensor bandwidths predicted by the step excitation at displacement are consistent with those predicted by the step bias changes, justifying the conventional procedure of evaluating the sensing bandwidth by the bias source. The sinusoidal tests were alternatively used to measure the sensor linearity and bandwidth. Compared to a single heater resistance, the model shows significant improvement in linearity for the differential sensor output, which is consistent with the experiment. In contrast to the bandwidth, the linearity is improved when using the shaped heaters in the electrothermal sensor rather than the straight ones. These results, which were observed in both static and dynamic simulations, agree well with the experiments and further validate the model. Compared to a constant bias voltage, both the model and the experiment show that the sensor bandwidth is reduced when deriving heaters with a constant current while the sensitivity is increased. As seen in Table II, the model also predicts that the differential sensor output is more linear when biasing the heaters with a constant current rather than a constant voltage.

\section{ACKNOWLEDGMENT}

The authors would like to thank A. Mohammadi and A. G. Fowler for their help in conducting the experiments reported in this work.

\section{REFERENCES}

[1] G. Binnig et al., "Ultrahigh-density atomic force microscopy data storage with erase capability," Appl. Phys. Lett., vol. 74, no. 9, pp. 1329-1331, Mar. 1999.

[2] C.-H. Kim and Y.-K. Kim, "Micro XY-stage using silicon on a glass substrate," J. Micromech. Microeng., vol. 12, no. 2, pp. 103-107, 2002.

[3] M. A. Lantz, G. K. Binnig, M. Despont, and U. Drechsler, "A micromechanical thermal displacement sensor with nanometre resolution," Nanotechnology, vol. 16, no. 8, pp. 1089-1094, 2005.

[4] Y.-C. Chen, R. T. M'Closkey, T. A. Tran, and B. Blaes, "A control and signal processing integrated circuit for the JPL-boeing micromachined gyroscopes," IEEE Trans. Control Syst. Technol., vol. 13, no. 2, pp. 286-300, Mar. 2005.

[5] L. Mol, G. de Graaf, L. Rocha, and R. Wolffenbuttel, "High-resolution capacitive measurement of microstructure displacement using coherent detection," in Proc. 19th Eur. Conf. Solid-State Transducers, Barcelona, Spain, Sep. 2005, pp. 1-4.

[6] J. Gorman, Y. Kim, and N. Dagalakis, "Control of MEMS nanopositioners with nano-scale resolution," in Proc. ASME Int. Mech. Eng. Congr. Exposit., Nov. 2006, pp. 151-159.

[7] L. Sun, J. Wang, W. Rong, X. Li, and H. Bao, "A silicon integrated micro nano-positioning XY-stage for nano-manipulation," J. Micromech. Microeng., vol. 18, no. 12, p. 125004, 2008.

[8] V. Todorov, V. Stavrov, and J. Kreuter, "Sub nm-resolution static measurement with MEMS displacement sensors," in Proc. Eng., vol. 25. 2011, pp. 591-594.

[9] G. Binnig and H. Rohrer, "The scanning tunneling microscope," Sci. Amer, vol. 253, no. 2, pp. 50-56, Aug. 1985.

[10] G. Binning, C. Quate, and C. Gerber, "Atomic force microscope," Phys. Rev. Lett., vol. 56, no. 9, pp. 930-933, Mar. 1986.

[11] A. Pantazi et al., "Control of MEMS-based scanning-probe data-storage devices," IEEE Trans. Control Syst. Technol., vol. 15, no. 5, pp. 824-841, Sep. 2007. 
[12] A. Pantazi, M. A. Lantz, G. Cherubini, H. Pozidis, and E. Eleftheriou, "A servomechanism for a micro-electro-mechanical-system-based scanning-probe data storage device," Nanotechnology, vol. 15, no. 10, p. S612, 2004.

[13] C. K. Pang et al., "Design, fabrication, sensor fusion, and control of a micro X-Y stage media platform for probe-based storage systems," Mechatronics, vol. 19, no. 7, pp. 1158-1168, 2009.

[14] Y. Zhu, S. O. R. Moheimani, and M. R. Yuce, "Simultaneous capacitive and electrothermal position sensing in a micromachined nanopositioner," IEEE Electron Device Lett., vol. 32, no. 8, pp. 1146-1148, Apr. 2011.

[15] P. Vettiger et al., "The 'millipede'-nanotechnology entering data storage," IEEE Trans. Nanotechnol., vol. 1, no. 1, pp. 39-55, Mar. 2002.

[16] E. Eleftheriou et al., "Millipede-A MEMS-based scanning-probe data-storage system," IEEE Trans. Magn., vol. 39, no. 2, pp. 938-945, Mar. 2003.

[17] M. A. Lantz, H. E. Rothuizen, U. Drechsler, W. Häberle, and M. Despont, "A vibration resistant nanopositioner for mobile parallel-probe storage applications," J. Microelectromech. Syst., vol. 16, no. 1, pp. 130-139, Feb. 2007.

[18] J. Chow and Y. Lai, "Displacement sensing of a micro-electro-thermal actuator using a monolithically integrated thermal sensor," Sensors Actuat. A, Phys., vol. 150, no. 1, pp. 137-143, 2009.

[19] B. Krijnen et al., "A single-mask thermal displacement sensor in MEMS," J. Micromech. Microeng., vol. 21, no. 7, p. 074007, 2011

[20] Y. Zhu, A. Bazaei, S. O. R. Moheimani, and M. R. Yuce, "Design, modeling, and control of a micromachined nanopositioner with integrated electrothermal actuation and sensing," J. Microelectromech. Syst., vol. 20, no. 3, pp. 711-719, Jun. 2011.

[21] A. Mohammadi, M. Yuce, and S. O. R. Moheimani, "Frequency modulation technique for MEMS resistive sensing," IEEE Sensors J., vol. 12, no. 8, pp. 2690-2698, Aug. 2012.

[22] A. Mohammadi, M. R. Yuce, and S. O. R. Moheimani, "A low-flicker-noise MEMS electrothermal displacement sensing technique," J. Microelectromech. Syst., vol. 21, no. 6, pp. 1279-1281, Dec. 2012.

[23] U. Dauderstädt, P. H. S. de Vries, R. Hiratsuka, and P. M. Sarro, "Silicon accelerometer based on thermopiles," Sens. Actuators A, Phys., vol. 46, nos. 1-3, pp. 201-204, 1995.

[24] G. K. Binnig, M. Despont, M. A. Lantz, and P. Vettiger, "Thermal movement sensor," U.S. Patent 7186019 , Mar. 6, 2007.

[25] A. Sebastian and D. Wiesmann, "Modeling and experimental identification of silicon microheater dynamics: A systems approach," J. Microelectromech. Syst., vol. 17, no. 4, pp. 911-920, Aug. 2008.

[26] A. Pantazi et al., "Probe-based ultrahigh-density storage technology," IBM J. Res. Develop., vol. 52, nos. 4-5, pp. 493-511, Jul. 2008.

[27] Y. Zhu, A. Bazaei, S. O. R. Moheimani, and M. Yuce, "A micromachined nanopositioner with on-chip electrothermal actuation and sensing," IEEE Electron Device Lett., vol. 31, no. 10, pp. 1161-1163, Oct. 2010.

[28] Y. Zhu, A. Bazaei, S. Moheimani, and M. R. Yuce, "Design, prototyping, modeling and control of a MEMS nanopositioning stage," in Proc. ACC, Jun./Jul. 2011, pp. 2278-2283.

[29] U. Dürig, "Fundamentals of micromechanical thermoelectric sensors," J. Appl. Phys., vol. 98, no. 4, p. 044906, 2005.

[30] A. G. Fowler, A. Bazaei, and S. O. R. Moheimani, "Design and analysis of nonuniformly shaped heaters for improved MEMS-based electrothermal displacement sensing," J. Microelectromech. Syst., vol. 22, no. 3, pp. 687-694, Jun. 2013.

[31] A. Bazaei, Y. Zhu, S. Moheimani, and M. R. Yuce, "Analysis of nonlinear phenomena in a thermal micro-actuator with a built-in thermal position sensor," IEEE Sensors J., vol. 12, no. 6, pp. 1772-1784, Jun. 2012.

[32] R. Legtenberg, A. W. Groeneveld, and M. Elwenspoek, "Comb-drive actuators for large displacements," J. Micromech. Microeng., vol. 6 , no. 3, pp. 320-329, 1996.

[33] J. L. Rodgers and W. A. Nicewander, "Thirteen ways to look at the correlation coefficient," Amer. Statist., vol. 42, no. 1, pp. 59-99, 1988.

[34] B. E. Deal and A. S. Grove, "General relationship for the thermal oxidation of silicon," J. Appl. Phys., vol. 36, no. 12, pp. 3770-3778, 1965.
[35] J. A. Kolodziej and T. Stręk, "Analytical approximations of the shape factors for conductive heat flow in circular and regular polygonal cross-sections," Int. J. Heat Mass Transf., vol. 44, no. 5, pp. 999-1012, 2001.

[36] A. Cowen, G. Hames, D. Monk, S. Wilcenski, and B. Hardy, SOIMUMPs Design Handbook, 8th ed. Park Durham, NC, USA: MEMSCAP Inc., 2011.

[37] E. Tannenbaum, "Detailed analysis of thin phosphorus-diffused layers in p-type silicon," Solid-State Electron., vol. 2, nos. 2-3, pp. 123-132, 1961.

[38] Y. K. Yong, A. G. Fowler, A. Mohammadi, and S. O. R. Moheimani, "Control of a MEMS nanopositioner for atomic force microscopy," in Proc. 6th IFAC Symp. Mech. Syst., Hangzhou, China, Apr. 2013.

[39] A. Mohammadi, A. G. Fowler, Y. K. Yong, and S. O. R. Moheimani, "A feedback controlled MEMS nanopositioner for on-chip high-speed AFM," IEEE J. Microelectromech. Syst., vol. 23, no. 3, pp. 610-619, Jun. 2014.

Ali Bazaei received the B.Sc. and M.Sc. degrees from Shiraz University, Shiraz, Iran, and the Ph.D. degrees from Tarbiat Modares University, Tehran, Iran, and the University of Western Ontario, London, ON, Canada, in 1992, 1995, 2004, and 2009, respectively, all in electrical engineering. From 1995 to 2000, he was an Instructor with Yazd University, Yazd, Iran. From 2004 to 2005, he was a Research Assistant with the Department of Electrical and Computer Engineering, University of Western Ontario. He is currently a Research Academic with the School of Electrical Engineering and Computer Science, University of Newcastle, Callaghan, NSW, Australia. His research interests include the general area of nonlinear systems, including the control and modeling of structurally flexible systems, friction modeling and compensation, neural networks, and microposition sensors.

S. O. Reza Moheimani (F'11) received the Ph.D. degree from the University of New South Wales, Canberra, NSW, Australia, in 1996.

He joined the University of Newcastle, Callaghan, NSW, in 1997, embarking on a new research program addressing the dynamics and control design issues related to high-precision mechatronic systems. He is the founder and Director of the Laboratory for Dynamics and Control of Nanosystems at the University of Newcastle, a multimillion-dollar state-of-the-art research facility. He has authored over 300 refereed papers and five books and edited volumes. His current research interests include ultrahigh-precision mechatronic systems, with particular emphasis on dynamics and control at the nanometer scale, including the applications of control and estimation in nanopositioning systems for high-speed scanning probe microscopy, modeling and control of microcantilever-based devices, control of microactuators in microelectromechanical systems, and design, modeling, and control of micromachined nanopositioners for on-chip atomic force microscopy.

Prof. Moheimani is a fellow of the International Federation of Automatic Control (IFAC) and the Institute of Physics, U.K. He was a recipient of the IFAC Nathaniel B. Nichols Medal in 2014, the IFAC Mechatronic Systems Award in 2013, the IEEE Control Systems Technology Award in 2009, the Australian Research Council Future Fellowship in 2009, the IEEE Transactions on Control Systems Technology Outstanding Paper Award in 2007, the Australian Research Council Post-Doctoral Fellowship in 1999, and several Best Student Paper Awards in various conferences. $\mathrm{He}$ has served on the Editorial Boards of a number of journals, including the IEEE/ASME Transactions on Mechatronics, the IEEE TransaCtions on CONTROL Systems TeChNology, and Control Engineering Practice. He is currently the Chair of the IFAC Technical Committee on Mechatronic Systems, and has chaired several international conferences and workshops. 